\title{
Small airway dysfunction is an independent dimension of wheezing disease in preschool children
}

\author{
Plamen Bokov ${ }^{1}$, Donies Masmoudi ${ }^{2}$, Flore Amat $^{3}$, Véronique Houdouin ${ }^{2}$, and Christophe \\ Delclaux $^{2}$ \\ ${ }^{1}$ Hopital Universitaire Robert-Debre \\ ${ }^{2}$ Robert-Debré Mother-Child University Hospital \\ ${ }^{3}$ Hopital Armand-Trousseau
}

March 24, 2021

\begin{abstract}
Background. Whether small airway dysfunction (SAD), which is prevalent in asthma, helps to characterize wheezing phenotypes is undetermined. The objective was to assess whether SAD parameters obtained from impedance measurement and asthma probability are linked. Methods. One hundred and thirty-nine preschool children (mean age 4.7 years, $68 \%$ boys) suffering from recurrent wheeze underwent impulse oscillometry that allowed calculating peripheral resistance and compliance of the respiratory system (markers of SAD) using the extended RIC model (central and peripheral Resistance, Inertance and peripheral Compliance of the respiratory system). Children were classified using the probability-based approach of GINA guidelines (few, some, most having asthma). A principal component analysis (PCA) that determined the dimensions of wheezing disease evaluated the links between SAD and asthma probability. Results. Forty-seven children belonged to the few, 28 to the some and 64 to the most having asthma groups. Whereas their anthropometrics and measured parameters were similar, the most having asthma group exhibited the lowest mean value of airway inertance after bronchodilator probably due to airway inhomogeneities. PCA characterized nine independent dimensions including a peripheral resistance (constituted by baseline peripheral resistance, AX, $\mathrm{R} 5-20 \mathrm{~Hz}, \mathrm{X} 5 \mathrm{~Hz}$ ), a central resistance (baseline central resistance, R20Hz) and an airway size dimension (post-bronchodilator inertance and central resistance). PCA showed that the SAD markers were independent from clinical dimensions (control and asthma probability were two other dimensions) and did not help to define wheezing phenotypes. Conclusions. Lung function parameters obtained from impulse oscillometry and asthma probability were belonging to independent dimensions of the wheezing disease.
\end{abstract}

Small airway dysfunction is an independent dimension of wheezing disease in preschool children

Plamen Bokov1, Donies Jallouli-Masmoudi2, Flore Amat3, Véronique Houdouin4 and Christophe Delclaux1 Running title: Small airway dysfunction in wheezers

1: Université de Paris, AP-HP, Hôpital Robert Debré, Service de Physiologie Pédiatrique-Centre du Sommeil, INSERM NeuroDiderot, F-75019 Paris, France

2: AP-HP, Hôpital Robert Debré, Service de Physiologie Pédiatrique-Centre du Sommeil, F-75019 Paris, France

3: AP-HP, Hôpital Robert Debré, Service de Pneumopédiatrie, INSERM UMR S 1136, F-75019 Paris, France

4: AP-HP, Hôpital Robert Debré, Service de Pneumopédiatrie, INSERM UMR S 976, F-75019 Paris, France

Correspondence: Pr Christophe Delclaux 
Service de Physiologie Pédiatrique

Hôpital Robert Debré

48, boulevard Sérurier

75019 Paris

Email : christophe.delclaux@aphp.fr

Word counts

Text : 2445; Abstract : 249

2 Tables, 2 Figures

Declarations of interest: none.

The study has not been founded

Abstract

Background. Whether small airway dysfunction (SAD), which is prevalent in asthma, helps to characterize wheezing phenotypes is undetermined. The objective was to assess whether SAD parameters obtained from impedance measurement and asthma probability are linked.

Methods. One hundred and thirty-nine preschool children (mean age 4.7 years, 68\% boys) suffering from recurrent wheeze underwent impulse oscillometry that allowed calculating peripheral resistance and compliance of the respiratory system (markers of SAD) using the extended RIC model (central and peripheral Resistance, Inertance and peripheral Compliance of the respiratory system). Children were classified using the probability-based approach of GINA guidelines (few, some, most having asthma). A principal component analysis (PCA) that determined the dimensions of wheezing disease evaluated the links between SAD and asthma probability.

Results. Forty-seven children belonged to the few, 28 to the some and 64 to the most having asthma groups. Whereas their anthropometrics and measured parameters were similar, the most having asthma group exhibited the lowest mean value of airway inertance after bronchodilator probably due to airway inhomogeneities. PCA characterized nine independent dimensions including a peripheral resistance (constituted by baseline peripheral resistance, AX, R5-20Hz, X5Hz), a central resistance (baseline central resistance, $\mathrm{R} 20 \mathrm{~Hz}$ ) and an airway size dimension (post-bronchodilator inertance and central resistance). PCA showed that the SAD markers were independent from clinical dimensions (control and asthma probability were two other dimensions) and did not help to define wheezing phenotypes.

Conclusions. Lung function parameters obtained from impulse oscillometry and asthma probability were belonging to independent dimensions of the wheezing disease.

Key words: airway compliance; airway resistance; asthma; impulse oscillometry; wheezing

Introduction

In a recent systematic review for the European Academy of Allergy and Clinical Immunology, the clinical practice recommendations on diagnostics of preschool wheeze stated that it is difficult to establish guidelines for monitoring asthma in preschool children.(1) Lung function measurement is an essential tool in the differential diagnosis of preschool wheezing, although reliable lung function measurements are challenging in this age group and the diagnosis value of functional tests remains debated.(1) This issue is important given the possible underestimation of asthma prevalence in preschool children.(2) On the other hand, wheeze has been associated with prescriptions of asthma medications in young children, which could lead to inappropriate and too high prescription rate of inhaled corticosteroids.(3) 
We recently showed that small airway dysfunction (SAD: increased peripheral resistance and decreased peripheral compliance of the respiratory system) was an almost constant finding in asthmatic children with increased interrupter resistance.(4) One may hypothesize that SAD markers obtained from a more sensitive method than spirometry, namely impulse oscillometry,(5-7) could be linked to asthma probability.

Several wheezing phenotypes coexist at preschool age but not all preschoolers with recurrent wheezing develop asthma at school-age; the asthma diagnosis still needs to be based on clinical predicted models or scores.(8) A probability-based approach, based on the pattern of symptoms during and between viral respiratory infections, is given in GINA guidelines that classifies the wheezing phenotypes of childhood.(9) We thus evaluated the ability of IOS indices to differentiate these wheezing phenotypes.

Methods

This cross-sectional study complied with The Strengthening the Reporting of Observational studies in Epidemiology (STROBE) guidelines. Consecutive preschool children aged 3 to 6 years referred for the first assessment of their wheezing disease were enrolled. These children were suffering from persistent wheeze (symptoms began before the age of 3 years and continued) or late-onset wheeze (symptoms began after the age of 3 years). The children were belonging to the "few" ( $<3$ episodes per year of symptoms [cough, wheeze, heavy breathing] for $<10$ days during upper respiratory tract infections and no symptoms between episodes) or "some" or "most" (>3 episodes per year of symptoms [cough, wheeze, heavy breathing] for $>$ 10 days during upper respiratory tract infections and between episodes symptoms) having asthma patterns described in GINA guidelines.(9) As compared to the group "some", the group "most" having asthma was defined by the presence of allergic sensitization or family history of asthma. Therapeutic steps (1 to 4$)$ and control assessment were those defined in GINA guidelines.(9) Adequate withdrawal of beta-agonist before testing was an additional inclusion criterion. Severe exacerbation definition was defined by the need of at least three days of oral steroid.

This study was approved by our local Ethics Committee (PHENOBS: N²018-430). The parents were informed of the collection of the prospective data for research purposes and they could request that their child to be exempted from this study in accordance with French law (non-interventional observational research).

Pulmonary function tests

Interrupter resistance (Rint) was measured using SpiroDyn'R apparatus (Dyn'R Ltd, Toulouse, France), as previously described.(4) Z-scores of Rint were calculated according to Merkus et al.(10)

Impedance of the respiratory system was measured using an impulse oscillatory system (IOS: Master Scope Body, Carefusion Technologies, Yorba Linda, California, USA), as previously described.(4) We used the following IOS variables: impedance at $5,10,15,20,25,30$ and $35 \mathrm{~Hz}$, resistance and reactance at $5 \mathrm{~Hz}$ and $20 \mathrm{~Hz}$, fall in resistance between R5 and R20 (R5-20Hz), area under the reactance curve (AX) and resonance frequency (Fres). Z-scores of IOS variables were calculated according to Gochicoa-Rangel et al.(11)

Rint and IOS measurements were obtained at baseline and after salbutamol (400 $\mu \mathrm{g}$ ) administration using an inhaler device.

We used two mechanistic models capable of accounting for significant frequency dependence of the respiratory impedance, which have previously been described.(4) Airway inertance Iaw is included in the RIC model as compared to the simple RC circuit (Resistance of the airways and Compliance of the alveoli). The RIC model with proximal shunting describes the effect of cheeks (proximal compliance) that could affect impedance measurement (measurement bias). In the extended RIC model, eRIC, R is partitioned in central $(\mathrm{Rc})$ and peripheral $(\mathrm{Rp})$ resistance of the respiratory system, while $\mathrm{Cp}$ is the peripheral compliance of the respiratory system (including parenchymal and chest wall compliances). Thus, SAD is characterized by Rp and $\mathrm{Cp}$. The model was fitted to the impedance data $(5-35 \mathrm{~Hz})$ and the minimization of a performance index allowed the calculation of model parameters, as previously done.(4) To determine the relative appropriateness of the various inverse model topologies, we used the corrected Akaike information criterion, as previously done.(4) 


\section{Statistical analyses}

Principal component analysis (PCA) is a mathematical procedure that uses an orthogonal transformation to convert a set of observations of possibly correlated variables into a set of values of uncorrelated variables called principal components (dimensions). We performed PCA of 27 variables (the minimal number of subjects providing usable data for the analysis should be five times the number of variables being analysed), using orthogonal varimax rotation. Results were expressed as mean \pm SD. Intergroup comparisons were made using t tests (two groups) or ANOVA (three groups), before and after salbutamol conditions were compared using paired t test. A P value $<0.05$ was deemed significant. Statistical analyses were performed with StatView 5.0 (SAS institute, Cary, North Carolina, USA) and OpenStat (version 5) softwares. Due to the exploratory design of the study, no correction for multiplicity of testing was done.(12)

Results

One hundred and sixty asthmatic children were enrolled of whom 12 children were excluded because the coherence of impedance measurements was unsatisfactory (coherence $5 \mathrm{~Hz}<0.60$ or $20 \mathrm{~Hz}<0.80$ ) and 9 children were excluded because their impedance spectra were better fitted by the RIC model with proximal (upper airway) shunting. The remaining 139 children who were adequately fitted by the extended RIC model are described in Table 1 (clinical characteristics) and Table 2 (functional characteristics). The Figure 1 shows the impedance spectra obtained by fitting the model. Whereas the results of IOS measurements were similar (almost normal lung function after bronchodilator), some modelled parameters were significantly different after bronchodilation (inertance and peripheral compliance) between the three groups. The most having asthma group exhibited both the highest value of mean peripheral compliance and the lowest mean value of airway inertance after bronchodilator. This group was also characterized by more frequent recent severe exacerbations.

Bronchodilator response based on $\mathrm{R} 5 \mathrm{~Hz}$ decrease correlated weakly with Rint response $(\mathrm{r}=0.20, \mathrm{p}=0.002)$ and with $\mathrm{Rp}$ response $(\mathrm{r}=0.15, \mathrm{p}=0.019)$, and mainly with $\mathrm{Rc}$ response $(\mathrm{r}=0.63, \mathrm{p}<0.0001)$. All modelled parameters, with the exception of Iaw, were significantly different after bronchodilator: $\mathrm{Rc}, \mathrm{p}=0.0001 ; \mathrm{Cp}$, $\mathrm{p}=0.0055 ; \mathrm{Rp}, \mathrm{p}=0.0096$.

Twenty-nine children were premature ([?]36 weeks of gestational age); they were characterized by increased baseline z-score of Rint $(2.05+-1.61$ versus $1.50+-1.23, \mathrm{p}=0.032)$, z-score of X5Hz $(0.68+-2.51$ versus $-0.28+-1.39, \mathrm{p}=0.002)$ and $\mathrm{Rp}(1.04+-0.43 \mathrm{kPa} . \mathrm{s} / \mathrm{L}$ versus $0.86+-0.28 \mathrm{kPa} . \mathrm{s} / \mathrm{L}, \mathrm{p}=0.003)$ as compared to non-premature children. After bronchodilation, the z-score of $\mathrm{X} 5 \mathrm{~Hz}(-0.55+-1.44$ versus $-1.43+-1.29$, $\mathrm{p}=0.0008)$ and $\mathrm{Rp}(0.95+-0.46 \mathrm{kPa} . \mathrm{s} / \mathrm{L}$ versus $0.77+-0.43 \mathrm{kPa} . \mathrm{s} / \mathrm{L}, \mathrm{p}=0.042)$ remained higher in premature children.

The Figure 2 shows the results of PCA using the 27 variables. Communality of all variables was $>60 \%$ (with the exception of post-BD peripheral resistance) and $73.8 \%$ of the total variance was explained by the factors (all factors had Eigenvalues $>1$ ). The Kaiser-Meyer-Olkin measure of sampling adequacy was 0.651.

Nine dimensions were found, some groupings of variables were expected such as anthropometrics (age, height: dimension 3), wheezing control (controlled versus uncontrolled and recent severe exacerbation: dimension 7) and asthma probability (wheezing patterns and therapeutic steps: dimension 4). Markers of SAD (peripheral resistance and compliance of the respiratory system) belonged to two different dimensions (dimension 1 and 8, respectively) that were independent from the clinical dimensions (wheezing control and asthma probability). A peripheral resistance (constituted by baseline peripheral resistance, $\mathrm{AX}, \mathrm{R} 5-20 \mathrm{~Hz}, \mathrm{X} 5 \mathrm{~Hz}$ and Rint, dimension 1), a central resistance (baseline central resistance and $\mathrm{R} 20 \mathrm{~Hz}$, dimension 2) and a postbronchodilator airway characterization (post-bronchodilator inertance, peripheral compliance and central resistance, dimension 5) were evidenced. The dimension 6 describes mainly full term boys, with elevated BMI z-score and early wheezing. The dimension 8 is constituted of children born prematurely of non-Caucasian origin.

Discussion 
The main result of our cross-sectional study in preschool wheezers is to show that lung function parameters obtained from impulse oscillometry and asthma probability were belonging to independent dimensions of the wheezing disease.

Previous classifications of wheezing phenotypes (such as episodic wheeze and multiple-trigger wheeze) do not appear to identify stable phenotypes,(13) and their clinical usefulness is uncertain, as stated in GINA 2020 guidelines.(9) These guidelines recommend the use of a probability-based approach.(9)

The studies evaluating the oscillometric technique's ability to classify lung function in wheezing and healthy children have been contradictory. Depending on the patient selection, both significant differences(14) and lack of differences $(15,16)$ have been reported. Subtle changes have been observed in the small airway indexes of IOS among children with mild to moderate recurrent wheezing.(17) Several studies have suggested that IOS parameters could be more sensitive than spirometry to diagnose asthma in children.(5,6) Finally, we recently showed that SAD was almost constant in young asthmatics exhibiting an increased interrupter resistance.(4) Based on this background, one may have supposed that IOS and additional modelling of SAD markers may have differentiated the three groups of wheezers defined on asthma-probability.

Among the three groups of wheezers, the most having asthma group was characterized by more recent severe exacerbations requiring oral steroid, which may seem expected in a group mainly constituted of asthmatic children. This group depicted the lower baseline interrupter resistance, which could be related to the higher proportion of asthmatics, responding to inhaled corticosteroid treatment (high dose for 59/64 children). IOS parameters were similar among the three groups, showing a slight mean basal impairment (for instance, mean z-score of $\mathrm{R} 5 \mathrm{~Hz}$ value of +1.46 in the whole population, with a mean z-score of post-bronchodilator of +0.12 , almost normal). Thus, in contradiction with our hypothesis, conventional IOS parameters were not able to differentiate these three wheezer groups, which may be related to the presence of asthmatic children into the three groups. Malmberg and colleagues showed better results for $\mathrm{R} 5 \mathrm{~Hz}$ and its bronchodilator response in identifying preschool children with probable asthma, but their groups were different from ours (treated asthma, probable asthma, chronic cough and healthy children).(15) By contrast, some parameters obtained from the eRIC model were significantly different among the three groups. Inertance is proportional to the length and inversely proportional to the cross-sectional area of the airways. The group with the highest probability of asthma had the smaller post-bronchodilator inertance; this result may seem counterintuitive since asthma has been associated with reduced peripheral airway caliber. This observation could be explained by the virtual decrease of inertance due to augmented airway inhomogeneities.(18) This group also had a higher compliance of the peripheral respiratory system that may favor ventilation inhomogeneity, a finding that has previously been evidenced in wheezing children having had a recent exacerbation.(19) This higher respiratory system compliance may have been related to either increased airway(20) or tissue compliance(21) that have been evidenced in asthma.

Reduced lung function in early infancy is predictive of persistent asthma in young adults and a persistent reduction in lung function, suggesting abnormal lung development and growth in utero or very early in life.(22) Preterm birth is associated with increased risks of asthma symptoms in childhood. The underlying mechanism seems to include persistently higher airway resistance.(23) We thus evaluated IOS measurements in premature versus non-premature children, showing that even after bronchodilation, the respiratory reactance and peripheral resistance remained higher in premature children, which further validate the parameters obtained from the eRIC model.

The main findings of our study are evidenced in Figure 2. We first confirm that AX, $\mathrm{R} 5-20 \mathrm{~Hz}$ and $\mathrm{X} 5 \mathrm{~Hz}$ are related to SAD since these parameters belonged to the same dimension than peripheral resistance of the respiratory system. Rint belonged to this dimension also, which is consistent with our previous results(4) and the previously reported high sensitivity of Rint in asthmatic children.(24) A proximal resistance dimension was also evidenced that included $\mathrm{R} 20 \mathrm{~Hz}$ and central resistance. Proximal and distal resistances were independent dimensions of wheezing disease. As previously evidenced in childhood asthma,(25) control, severity (treatment) and lung function were independent dimensions of the wheezing disease also. The ATLANTIS study showed that SAD is present across adult patients with all severities of asthma.(26) In this latter study, 
the levels of statistical correlations between IOS parameters and severity or control were mild (maximum r value of 0.25 and 0.23 for the number of exacerbations and GINA score, respectively).(26) Thus, our results in wheezing children are in agreement with those obtained in adult asthmatics. Asthma probability was an independent dimension of wheezing disease, which may explain the difficulty to predict asthma in this population. $(8,9)$ Importantly, SAD markers were not linked to this asthma probability.

One may have hypothesized that an increased airway tone (reduced compliance) would have been associated with reduced airway caliber (increased resistance). Nevertheless, peripheral compliance and resistance belonged to two independent dimensions (dimensions 8 and 1, respectively). Peripheral resistance is related to both airway anatomy and smooth muscle contraction, which may explain the absence of correlation. Reduced peripheral airway caliber in asthmatics is well known.(27) Moreover, both a decrease and an increase in airway distensibility have been described in asthma. $(20,28)$ It has to be highlighted that peripheral compliance of the respiratory system is related to airway but also tissue compliance. Increased pulmonary compliance has recently been described in some asthmatic children that is associated with increased static volumes.(21)

The other dimensions that were evidenced further suggest the validity of the PCA. Gestational age was linked to ethnicity in the ninth dimension, a fact that is well demonstrated.(29) The sixth dimension regrouped sex, z-score of BMI and persistent wheezing that could be in agreement with the "fat happy wheezer" phenotype in boys. (30)

Our study has some limitations. The included children were unable to perform spirometry, whether the children with abnormal Rint or IOS parameters had normal or abnormal spirometry cannot be assessed. Given the absence of follow-up of the children, asthma diagnosis cannot be assessed confidently since bronchodilator reversibility may be observed in other children during their follow-up. Nevertheless, our study was designed to assess the usefulness of the cross-sectional assessment by IOS parameters, which belonged to other dimensions than the clinical ones.

In conclusion, the characterization of SAD by IOS gives parameters independent from clinical dimensions in wheezing preschool children that does not help to define wheezing phenotypes of Global Initiative for Asthma guidelines in a cross-sectional design.

Acknowledgments

The authors thank the nurses of the Respiratory physiology unit of Robert Debre hospital for expert technical assistance.

Impact statement, Key Message

Impulse oscillometry does not help to predict asthma in wheezing preschool children. Lung function is an independent dimension of wheezing disease, as shown in asthma.

Author Contributions

Conceptualization: PB and CD2; Formal analysis: PB, CD2; Investigation: PB, DM; Methodology: PB, FA, VH; Project administration: CD2, FA, VH; Supervision: CD2, FA, VH; Validation: PB, CD2; Roles/Writing - original draft: PB, CD2; Writing - review \&editing: DM, FA, VH.

\section{References}

1. Elenius V, Chawes B, Malmberg PL et al. Lung function testing and inflammation markers for wheezing preschool children: A systematic review for the EAACI Clinical Practice Recommendations on Diagnostics of Preschool Wheeze. Pediatr Allergy Immunol Published Online First: 22 November 2020. doi:10.1111/pai.13418

2. Lawson JA, Brozek G, Shpakou A et al. An international comparison of asthma, wheeze, and breathing medication use among children. Respir Med 2017: 133:22-28. 
3. Mikalsen IB, Dalen I, Karlstad O et al. Airway symptoms and atopy in young children prescribed asthma medications: A large-scale cohort study. Pediatr Pulmonol 2019: 54:1557-1566.

4. Bokov P, Bafunyembaka G, Medjahdi N et al. Cross-sectional phenotyping of small airway dysfunction in preschool asthma using the impulse oscillometry system. J Asthma 2020: :1-13.

5. Marotta A, Klinnert MD, Price MR et al. Impulse oscillometry provides an effective measure of lung dysfunction in 4-year-old children at risk for persistent asthma. J Allergy Clin Immunol 2003: 112:317-322.

6. Komarow HD, Skinner J, Young M et al. A study of the use of impulse oscillometry in the evaluation of children with asthma: analysis of lung parameters, order effect, and utility compared with spirometry. Pediatr Pulmonol 2012: 47:18-26.

7. Song TW, Kim KW, Kim ES et al. Utility of impulse oscillometry in young children with asthma. Pediatr Allergy Immunol Off Publ Eur Soc Pediatr Allergy Immunol 2008: 19:763-768.

8. Castro-Rodriguez JA, Cifuentes L, Martinez FD. Predicting Asthma Using Clinical Indexes. Front Pediatr 2019: 7:320.

9. Reports. Glob. Initiat. Asthma - GINA. https://ginasthma.org/reports/ (accessed 11 Sep2020).

10. Merkus PJFM, Stocks J, Beydon N et al. Reference ranges for interrupter resistance technique: the Asthma. UK Initiat Eur Respir J 2010: 36:157-163.

11. Gochicoa-Rangel L, Del Rio-Hidalgo R, Hernandez-Ruiz J et al. Validating Reference Equations for Impulse Oscillometry in Healthy Mexican Children. Respir Care 2017: 62:1156-1165.

12. Rothman KJ. No adjustments are needed for multiple comparisons. Epidemiology 1990: 1:43-46.

13. Raaymakers MJA, Brand PLP, Landstra AM et al. Episodic viral wheeze and multiple-trigger wheeze in preschool children are neither distinct nor constant patterns. A prospective multicenter cohort study in secondary care. Pediatr Pulmonol 2019: 54:1439-1446.

14. Oostveen E, Dom S, Desager K et al. Lung function and bronchodilator response in 4-year-old children with different wheezing phenotypes. Eur Respir J 2010: 35:865-872.

15. Malmberg LP, Pelkonen AS, Haahtela T et al. Exhaled nitric oxide rather than lung function distinguishes preschool children with probable asthma. Thorax 2003: 58:494-499.

16. Cuijpers CE, Wesseling GJ, Kessels AG et al. Low diagnostic value of respiratory impedance measurements in children. Eur Respir J 1997: 10:88-93.

17. Knihtila H, Kotaniemi-Syrjanen A, Pelkonen AS et al. Small airway function in children with mild to moderate asthmatic symptoms. Ann Allergy Asthma Immunol Off Publ Am Coll Allergy Asthma Immunol 2018: 121:451-457.

18. Lutchen KR, Hantos Z, Petak F et al. Airway inhomogeneities contribute to apparent lung tissue mechanics during constriction. J Appl Physiol Bethesda Md 1985 1996: 80:1841-1849.

19. Kowalik K, Dai R, Safavi S et al. Persistent ventilation inhomogeneity after an acute exacerbation in preschool children with recurrent wheezing. Pediatr Allergy Immunol Off Publ Eur Soc Pediatr Allergy Immunol 2020: 31:608-615.

20. Al-Alwan A, Bates JHT, Chapman DG et al. The nonallergic asthma of obesity. A matter of distal lung compliance. Am J Respir Crit Care Med 2014: 189:1494-1502.

21. Comberiati P, Spahn JD, Paull K et al. Lung mechanical properties distinguish children with asthma with normal and diminished lung function. Clin Exp Allergy J Br Soc Allergy Clin Immunol 2020: 50:453462. 
22. Owens L, Laing IA, Zhang $\mathrm{G}$ et al. Infant lung function predicts asthma persistence and remission in young adults. Respirol Carlton Vic 2017: 22:289-294.

23. Sonnenschein-van der Voort AMM, Gaillard R, de Jongste JC et al. Foetal and infant growth patterns, airway resistance and school-age asthma. Respirol Carlton Vic 2016: 21:674-682.

24. Merkus PJ, Mijnsbergen JY, Hop WC et al. Interrupter resistance in preschool children: measurement characteristics and reference values. Am J Respir Crit Care Med 2001: 163:1350-1355.

25. Mahut B, Peyrard S, Delclaux C. Exhaled nitric oxide and clinical phenotypes of childhood asthma. Respir Res 2011: 12:65.

26. Postma DS, Brightling C, Baldi S et al. Exploring the relevance and extent of small airways dysfunction in asthma (ATLANTIS): baseline data from a prospective cohort study. Lancet Respir Med 2019: 7:402-416.

27. Martinez FD. Early-Life Origins of Chronic Obstructive Pulmonary Disease. N Engl J Med 2016: $375: 871-878$.

28. Kelly VJ, Brown NJ, Sands SA et al. Effect of airway smooth muscle tone on airway distensibility measured by the forced oscillation technique in adults with asthma. J Appl Physiol Bethesda Md 1985 2012: 112:1494-1503.

29. Manuck TA. Racial and ethnic differences in preterm birth: A complex, multifactorial problem. Semin Perinatol 2017: 41:511-518.

30. Turner S, Zhang G, Young S et al. Associations between postnatal weight gain, change in postnatal pulmonary function, formula feeding and early asthma. Thorax 2008: 63:234-239.

Table 1. Clinical characteristics of the wheezing children.

Characteristics Whole population Few having asthma Some having asthma Most having asthma P value Intergroup

comparisons

Number of children 139472864

ethnicity, C / B / A / M 90/37/6/6 32/13/1/1 16/9/1/2 42/15/4/3 0.797

gestational age, weeks $38+-338+-338+-438+-20.500$

sex (male, \%) 94 (68) 34 (72) 18 (64) 42 (66) 0.692

age, years $4.7+-0.84 .7+-0.84 .7+-0.84 .7+-0.80 .926$

height, cm $108.3+-7.4108 .7+-8.3107 .7+-5.8108 .2+-7.40 .700$

weight, $\mathrm{kg} 19.3+-3.919 .6+-4.418 .7+-3.319 .3+-3.90 .584$

BMI z-score $0.40+-1.330 .44+-1.420 .18+-1.480 .47+-1.200 .542$

persistent wheeze, n (\%) 101 (73) 32 (68) 23 (82) 46 (72) 0.410

personal atopy (positive skin prick test), (n tested) 53 (120) 13 (33) 040 (59) ND

rhinitis, n (n available) 45 (129) 12 (43) 5 (28) 28 (58) $0.0111,2<3$

eczema, n (n available) 49 (124) 17 (40) 4 (28) 28 (56) $0.0062<1,3$

parental asthma, n (n available) 50 (130) 12 (41) 038 (61) ND

parental atopy, n (n available) 58 (114) 12 (37) 12 (27) 34 (50) $0.0031,2<3$

Asthma control 
GINA score, 0 / 1 / 2 / 3 / 4 (past month) 64/20/24/17/14 22/8/5/6/6 16/4/4/3/1 26/8/15/8/7 0.655

controlled / uncontrolled, n 64/75 22/25 16/12 26/38 0.340

severe exacerbation within last three months, n (\%) 39 (28) 10 (21) 4 (14) 25 (39) 0.023

Asthma treatment

beta-agonist on demand only (inhaled treatment), n 484110 ND 2=3

low ICS dose, n 2011 ND 2=3

medium ICS dose, n 7034 ND $2=3$

high ICS dose, n 8202359 ND 2=3

LABA, n 4201131 ND 2=3

LTRA, n 2401014 ND 2=3

Therapeutic steps

$1 / 2$ / 3 / 4 47/6/47/39 47/0/0/0 0/2/17/9 0/4/30/30 ND

BMI denotes body mass index, BD denotes bronchodilator, ICS notes inhaled corticosteroid, LABA denotes long-acting beta-agonist, LTRA denotes leukotriene receptor antagonist

Ethnicity: C denotes Caucasian, B denotes African-American, A denotes Asian and M denotes mixed

Results are provided as mean +- SD or absolute number with percentage (\%)

Table 2. Functional characteristics of the wheezing children.

Characteristics Whole population Few having asthma Some having asthma Most having asthma P value Intergroup

comparisons

Number of children 139472864

Resistance and impedance measurements

Rint, z-score before BD 1.49 +- 1.301 .83 +- $1.361 .69+-1.361 .14+-1.160 .0331,2>3$

Rint, z-score after BD 0.06 +- 1.100 .24 +- 1.150 .39 +- $1.24-0.21$ +- 0.930 .054

Coherence 5Hz, before BD $0.76+-0.080 .78+-0.090 .75+-0.080 .75+-0.070 .141$

Coherence 20Hz, before BD $0.94+-0.040 .94+-0.050 .94+-0.040 .94+-0.040 .977$

Coherence 5Hz, after BD $0.73+-0.070 .74+-0.080 .72+-0.080 .72+-0.070 .444$

Coherence 20Hz, after BD $0.92+-0.040 .92+-0.040 .92+-0.040 .92+-0.040 .846$

R20Hz, z-score before BD $1.08+-1.650 .92$ +- 1.281 .21 +- $1.911 .215+-1.780 .706$

R20Hz, z-score after BD $0.39+-1.520 .20$ +- $1.690 .59+-1.610 .45$ +- 1.350 .517

R5Hz, z-score before BD 1.46 +- 2.131 .16 +- $1.831 .93+-2.721 .47$ +- 2.040 .245

$\mathrm{R} 5 \mathrm{~Hz}, \mathrm{z}$-score after BD 0.12 +- $1.60-0.07$ +- 1.680 .54 +- 1.720 .08 +- 1.480 .269

R5-20Hz, z-score before BD $1.01+-2.310 .75+-2.081 .54+-2.850 .96+-2.210 .261$

R5-20Hz, z-score after BD -0.24 +- $1.45-0.33+-1.360 .17+-1.50-0.37+-1.480 .234$

$\mathrm{X} 5 \mathrm{~Hz}, \mathrm{z}$-score before BD -0.28+- $1.58-0.12+-1.46-0.22+-1.66-0.41+-1.630 .838$ 
$\mathrm{X} 5 \mathrm{~Hz}, \mathrm{z}$-score after BD -1.60 +- $1.29-1.52+-1.55-1.45+-1.22-1.74+-1.100 .525$

AX, z-score before BD $0.78+-2.310 .73+-2.540 .79+-1.820 .81+-2.360 .722$

AX, z-score after BD -0.87 +- $1.44-0.85+-1.55-0.43+-1.37-1.09+-1.370 .136$

Fres, z-score before BD 0.10 +- $1.80-0.12$ +- $1.40-0.06+-2.060 .33+-1.940 .144$

Fres, z-score after BD -0.91 +- $1.29-0.99+-1.23-0.45+-1.46-1.06+-1.240 .102$

eRIC model indices

Performance Index before BD 0.045 +- 0.0560 .046 +- 0.0620 .061 +- $0.0710 .037+-0.0420 .169$

corrected AIC before BD -70 +- 11 -70 +- 11 -66 +- 12 -72 +- 110.066

Performance Index after BD 0.041 +- 0.0540 .033 +- 0.0240 .042 +- 0.0450 .047 +- 0.0710 .439

corrected AIC after BD -72 +- $12-72$ +- 9 -70 +- 11 -72 +- 140.752

Rc, kPa.s/L before BD 0.75 +- 0.160 .74 +- 0.140 .76 +- 0.160 .75 +- 0.170 .872

Rc, kPa.s/L after BD 0.70 +- 0.160 .69 +- 0.170 .70 +- 0.140 .71 +- 0.160 .738

Iaw, cPa.s2/L before BD 111 +- 42110 +- 41112 +- 46112 +- 410.986

Iaw, cPa.s2/L after BD 116 +- 36124 +- 32122 +- 33108 +- 400.044 1,2>3

Cp, mL/kPa before BD 84 +- 13591 +- 15187 +- 12975 +- 1130.769

$\mathrm{Cp}, \mathrm{mL} / \mathrm{kPa}$ after BD 108 +- 14774 +- 7290 +- $156141+-1850.0401,2<3$

Rp, kPa.s/L before BD 0.93 +- 0.290 .98 +- $0.290 .93+-0.290 .90+-0.280 .363$

Rp, kPa.s/L after BD $0.81+-0.530 .79+-0.450 .87+-0.520 .80+-0.600 .829$

Bronchodilator response, $\%$

$\mathrm{R} 5 \mathrm{~Hz}$ decrease, $\%$ baseline $14+-1514$ +- 1613 +- 1315 +- 160.606

Results are provided as mean +- SD. BD denotes bronchodilator. AIC denotes Akaike Information Criterion

Results in italic are quality criteria of impedance measurement or model fitting

Figure legends

Figure 1. The impedance spectra obtained by fitting the model.

Respiratory system resistance (A) and reactance (B) obtained by fitting the model (thick solid lines) to the prebronchodilator data of the experimental measurements represented by the median values and the 25th and 75 th percentiles for each frequency.

Figure 2. Results of the principal component analysis.

PCA shows factors which have very high (red to brown, positive values or blue colors, negative values) or very low (green color) loadings for the original variables and thus simplifies the interpretation of the resulting factors. Each single dimension is characterized by the variables with high loadings (red and blue colors).

The 27 variables were: 1 ethnicity (0 Caucasian), 2 age, 3 sex (0 female), 4 height, 5 z-score of BMI, 6 gestational age, 7 persistent wheezing ( 0 absent, 1 present), 8 severe exacerbation within previous 3 months (0 absent, 1 present), 9 GINA therapeutic steps (1 to 4), 10 probability ( 1 few, 2 some, 3 most having asthma), 11 control ( 0 controlled versus 1 uncontrolled based on GINA score), 12 baseline Rint, 13 baseline Fres, 14 baseline AX, 15 baseline R5Hz, 16 baseline X5Hz, 17 baseline R20Hz, 18 baseline R5-20Hz, 19 baseline Rc, 20 baseline Iaw, 21 baseline Cp, 22 baseline Rp, 23 post-BD Rc, 24 post-BD Iaw, 25 post-BD 
Cp, 26 post-BD Rp, $27 \mathrm{BD}$ response based on $\mathrm{R} 5 \mathrm{~Hz}$. Raw values of pulmonary function parameters were used in the PCA.

SAD denotes small airway dysfunction, BD denotes bronchodilator.
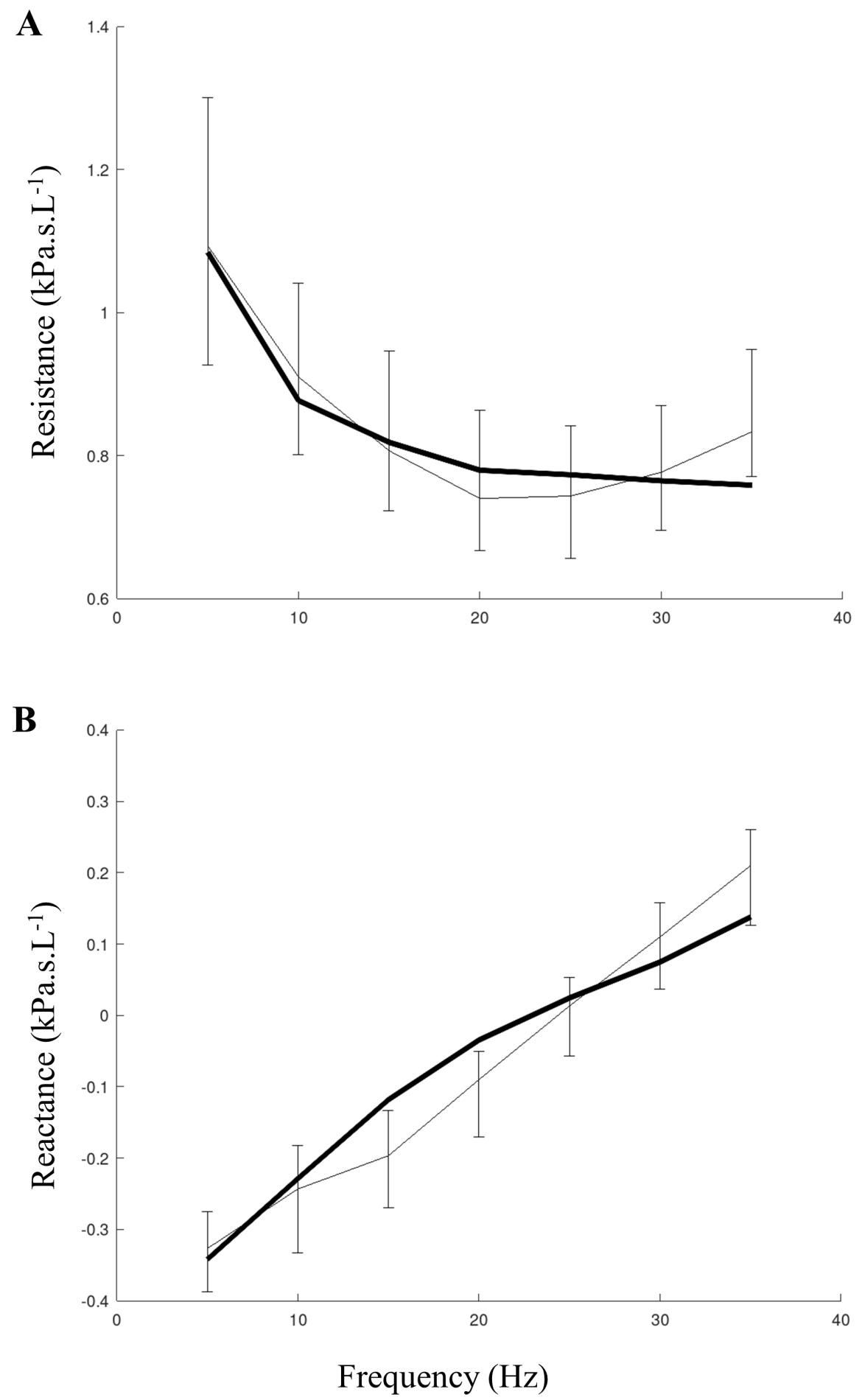


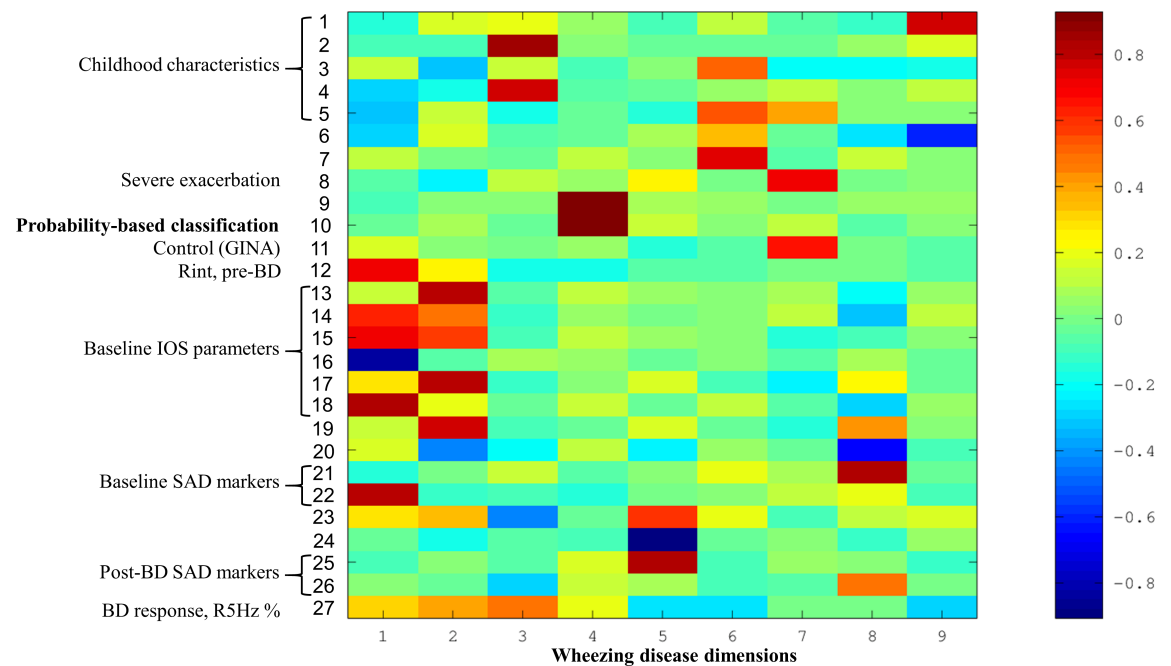

